\title{
THE EFFECT OF FATIGUE ON KINEMATICS AND KINETICS OF BASKETBALL DRIBBLING WITH CHANGES OF DIRECTION
}

\author{
Feng Li, Tomislav Rupčić, and Damir Knjaz \\ University of Zagreb, Faculty of Kinesiology, Laboratory for Sports Games, Croatia
}

Original scientific paper

DOI $10.26582 / \mathrm{k} .53 .2 .12$

\begin{abstract}
:
Basketball dribbling is one of the key elements in basketball game. There is a lack of studies investigating the effect of fatigue on kinematics and kinetics in basketball dribbling. There are two primary aims of this study: (1) to explore the effect of fatigue on kinematics and kinetics in dribbling with the change of directions; (2) to determine the effect of fatigue on dribbling speed. Fourteen Croatian senior male basketball players (age: $21.16 \pm 3.43$ years; body height: $188.81 \pm 6.88 \mathrm{~cm}$; body mass: $87.81 \pm 6.06 \mathrm{~kg}$; body fat: $13.34 \pm 3.52 \%$ ), not power forwards or centers, participated in the study. Each player performed two types of change of direction (COD) while dribbling: front COD and spin move in the non-fatigued and then in the fatigued state. Xsens suit and Novel insoles were used to measure the kinematic and kinetic parameters. In terms of the front $\mathrm{COD}$, the results of this study demonstrated that the maximum angular velocity in the knee $(\mathrm{p}=.028)$ and wrist joint $(\mathrm{p}=.007)$ as well as maximum force $(\mathrm{p}=.004)$ significantly decreased in the fatigued state. In terms of the spin move, the results showed that there were significant differences in pelvis velocity $(p=.000)$, the maximum angular velocity in the knee joint $(\mathrm{p}=.020)$, and the first step velocity $(\mathrm{p}=.010)$ between the fatigued and non-fatigued states. No significant difference was found in the pelvis position, minimum angle in the knee joint and maximum force. Importantly, dribbling speed significantly decreased in the fatigued state $(p=.002)$. The findings of this study suggest that coaching staff should design appropriate training programs to optimize players' ability to resist fatigue when dribbling under real game speed conditions.
\end{abstract}

Key words: dribble, change of direction, spin move, velocity, angular velocity, joint angle

\section{Introduction}

During the competition, there are three essential action options for the next movement when a player holds the ball — he/she may dribble, pass, or take a shot (Arias-Estero, 2013). In a set offense, a player with a proficient dribbling technique is able to break the opponent's intensive defense (e.g., using a crossover, penetration, and spin move), which creates free space to pass the ball to his/ her teammates thus creating an open shot opportunity for them (Arias, Argudo, \& Alonso, 2012; Arias-Estero, 2013), or to penetrate to the restricted area (paint). Additionally, it has been previously observed that fast break (Christmann, Akamphuber, Müllenbach, \& Güllich, 2018; Conte, Favero, Niederhausen, Capranica, \& Tessitore, 2017; Evangelos, Alexandros, \& Nikolaos, 2005; Matulaitis \& Bietkis, 2021) and transition to offense (Matulaitis \& Bietkis, 2021; Milanović, Selmanović, \& Škegro, 2014) are the most scoring efficient modes of attacking, both of which require high-speed dribbling to provide a temporal-spatial advantage over the defender while driving to the basket (Conte, et al.,
2017). Moreover, Conte, Favero, Niederhausen, Capranica, and Tessitore (2016) have pointed out that the proper technique of passing and dribbling reduces the number of turnovers and induces more assists (Arias, et al., 2012). Therefore, it can be said that the effective dribbling technique plays an important role in determining the outcome of a match. When it comes to dribbling in basketball, one can recognize dribbling in place, dribbling in a straight line, dribbling with a change-of-pace, and dribbling with a change of direction (COD) (Krause \& Nelson, 2018). Furthermore, it has been stated that dribbling with COD is the most frequently used way of dribbling during the competition (Cortis, et al., 2011; Fujii, Yamada, \& Oda, 2010).

Given the importance of dribbling technique, various aspects have been investigated so far (Dos Santos, Pacheco, Basso, Bastos, \& Tani, 2020; Guimarães, et al., 2019; Robalo, Diniz, Fernandes, $\&$ Passos, 2021). A number of studies have investigated the frequency and efficiency of dribbling in basketball games and have reported that dribbling skills are constantly used during basketball games 
with elite players dribbling during $\sim 10 \%$ of the live time (Andrić, 2011; Scanlan, Dascombe, \& Reaburn, 2011; Scanlan, Dascombe, Kidcaff, Peucker, \& Dalbo, 2015). In addition, some other aspects of dribbling skills have been investigated such as skill improvement (Dos Santos, et al., 2020; Fujii, et al., 2010), technique evaluation (Conte, et al., 2020; Jakovljević, Karalejić, Ivanović, Štrumbelj, \& Erčulj, 2017; Robalo, et al., 2021), and the effect of supplementation on dribbling performance (Scanlan, et al., 2019). Surprisingly, literature revealed few studies detecting the influence of fatigue on basketball dribbling performance.

Basketball is an intermittent high-intensity team sport characterized by short sprints, abrupt jumps, shufflings, and CODs, which can lead to acute and accumulated chronic fatigue (Erčulj, Blas, \& Bračič, 2010; Mancha-Triguero, GarcíaRubio, Calleja-González, \& Ibáñez, 2019; Li, Knjaz, \& Rupčić, 2021; Mancha-Triguero, GarcíaRubio, Gamonales, \& Ibáñez, 2021). It is well understood that fatigue has a negative influence on players' performance (Calleja-González, et al., 2016; Erculj \& Supej, 2009; Mulazimoglu, Yanar, Tunca Evcil, \& Duvan, 2017; Thorpe, Atkinson, Drust, \& Gregson, 2017). Several studies observed the effect of different physiological loads on shooting performance, reporting that shooting accuracy significantly decreased (Erculj \& Supej, 2009; Rupčić, et al., 2020). Similarly, the passing performance has been investigated by several studies, showing that passing accuracy decreased when players were in the fatigued state ( $\mathrm{Li}$, et al., 2021; Lyons, Al-Nakeeb, \& Nevill, 2006). Consequently, it is specifically important that players maintain a high level of skill performance under the influence of fatigue in order to win a game (Conte, et al., 2017).

With the development of technology, many researchers have used motion capture systems to objectively analyze basketball players' skill execution (Erculj \& Supej, 2009; Nakano, Fukashiro, \& Yoshioka, 2020; Okazaki \& Rodacki, 2012; Okubo \& Hubbard, 2015; Uygur, Goktepe, Ak, Karabörk, \& Korkusuz, 2010). In the past, researchers have shown an increased interest in the influence of fatigue on kinematics of basketball skills (Erculj \& Supej, 2009; Uygur, et al., 2010). Erculj and Supej (2009) observed the influence of fatigue on kinematics of shooting. Their findings revealed that the position of the release arm and shoulder significantly changed when players were shooting under the moderate- and high-intensity fatigue conditions (Erculj \& Supej, 2009). Uygur et al. (2010) found that fatigue did not affect selected kinematic variables of the free throw. To the best of the author's knowledge, however, no previous study has investigated the influence of fatigue on kinematics of basketball dribbling.
Therefore, there were two primary aims of this study: (1) to determine the effect of fatigue on kinematics and kinetics of dribbling with COD and (2) to observe the effect of fatigue on speed of dribbling with COD. It was hypothesized that differences in kinematic and kinetic parameters as well as in dribbling speed would be observed between dribblings of both types performed in the non-fatigued and fatigued state.

\section{Methods}

\section{Participants}

Fourteen Croatian senior male basketball players (age: 21.16 \pm 3.43 years; body height: $188.81 \pm 6.88 \mathrm{~cm}$; body mass: $87.81 \pm 6.06 \mathrm{~kg}$; body fat percentage: $13.34 \pm 3.52 \%$; basketball experience: $9.42 \pm 4.14$ years) from three professional basketball clubs volunteered to participate in this study. Inclusion criteria were a regular participation in practice sessions and competitions, and the absence of any injury in the past six months. The additional inclusion criterion was playing in the positions of either point guards $(n=5)$, shooting guards $(n=4)$, or small forwards $(\mathrm{n}=5)$ since basketball is a game in which players have position-specific roles according to which outside players should have a high level of fitness related to dribbling (Sekulic, et al., 2017). The exclusion criterion was playing in the positions of power forwards and centers. Players refrained from heavy training for at least one day preceding testing sessions. Prior to the testing, participants were fully informed of the study protocol and provided a written informed consent. The obtained data were treated with the greatest confidentiality and scientific rigor as their use was being restricted by the guidelines for research projects following the scientific method required in each case, complying with the Organic Law 15/1999 of the 13th of December on the Protection of Personal Data (OLPPD); the proceedings used respected the ethic criteria of the Responsible Committee of Human Experimentation and the Helsinki Statement of 2008, updated in Fortaleza, October 2013 (2013 version). All testing procedures were approved by the Ethics Committee of the Faculty of Kinesiology, University of Zagreb (ethical approval number: 108/2020), according to the ethical standards of the Declaration of Helsinki.

\section{Experimental procedures}

\section{Instruments}

In order to monitor players' fatigue levels, blood lactate (BL) concentration measurements were conducted using a portable lactate analyzer (Lactate Scout 3, manufacturer: SensLab GmbH, Leipzig, Germany) at four time points: before warm-up, after warm-up, after the first testing, and after the fatigue protocol. Additionally, heart rate (HR) was 
monitored throughout the testing by a heart rate monitor (Polar H10, manufacturer: Polar, Kempele, Finland). The reliability and validity of Lactate Scout 3 and Polar H10 were previously confirmed (Belić, et al., 2016; Hinde, White, \& Armstrong, 2021; Speer, Semple, Naumovski, \& Mckune, 2020; Tanner, Fuller, \& Ross, 2010).

Kinematic parameters were measured using the Xsens MVN inertial system (Xsens Technologies B.V., Enschede, The Netherlands). The players wore a full-body suit equipped with 17 wireless motion trackers (sampling frequency of $60 \mathrm{~Hz}$ ) to ensure a full 3D motion analysis. The kinematic parameters were derived from the corresponding MVN Studio BIOMECH software (Xsens Technologies B.V., Enschede, The Netherlands). A previous study has verified the reliability and validity of the Xsens kinematic suit for the kinematic analysis of basketball skills (Robert-Lachaine, Mecheri, Larue, \& Plamondon, 2017). In addition, it was used in previous studies for measuring similar data on the basketball court (Li, et al., 2021; Slawinski, et al., 2018).

For kinetic analysis pressure insoles were inserted in the participants' shoes for pressure detection with the sampling rate of $100 \mathrm{~Hz}$ (Novel Pedar model W, Germany). Insoles are thin and light $(2 \mathrm{~mm})$, having minimal influence on players' performance during testing, which is particularly important during a very dynamic COD in dribbling. Data were derived from the corresponding Novel software (Loadsol analysis 25.3.6). Previous studies have confirmed the reliability and validity of the Novel pressure insoles for analyzing foot pressure in sports (Sorrentino, et al., 2020; Stricker, Scheiber, Lindenhofer, \& Müller, 2010). The standard calibration of pressure insoles was performed according to the manufacturers' instructions (Novel GmBh, Munich).

Players were asked to dribble and change direction as fast as possible. Their time was recorded by photocells (WittyGate, Microgate, Bolzano, Italy). The reliability and validity of photocells were proved and used by previous studies (Balsalobre-Fernández, et al., 2019; Doyle, Browne, \& Horan, 2020).

\section{Protocol}

This study used a repeated measurements study design - measurements of the kinetic and kinematic parameters of CODs in dribbling were conducted first in the non-fatigued and then in the fatigued state. All the fourteen players underwent the same protocol: they had one day of rest before the testing that consisted of the following: warm-up, a non-fatigued dribbling test, a fatigue protocol, and a fatigued dribbling test.

Prior to the testing procedure, basic anthropometric measurement was executed and data were used for the systems calibration, performed according to the instruction of the manufacturer (Xsens Technologies B.V., Netherlands). In order to ensure that all the participants were familiarized with the testing protocol, the warm-up consisted of five minutes of jogging, five minutes of dynamic stretching, and five minutes of low intensity dribbling consistent with the testing protocol. Afterwards, the calibration was conducted according to the manufacturers' instructions and the players were asked to stand in $\mathrm{N}$-pose. After the calibration and synchronization, the players were asked to abduct their arms (up to first approx. $90^{\circ}$ and then $180^{\circ}$ ) to ensure that the system was correctly calibrated, after which the dribbling testing commenced. In order to synchro-

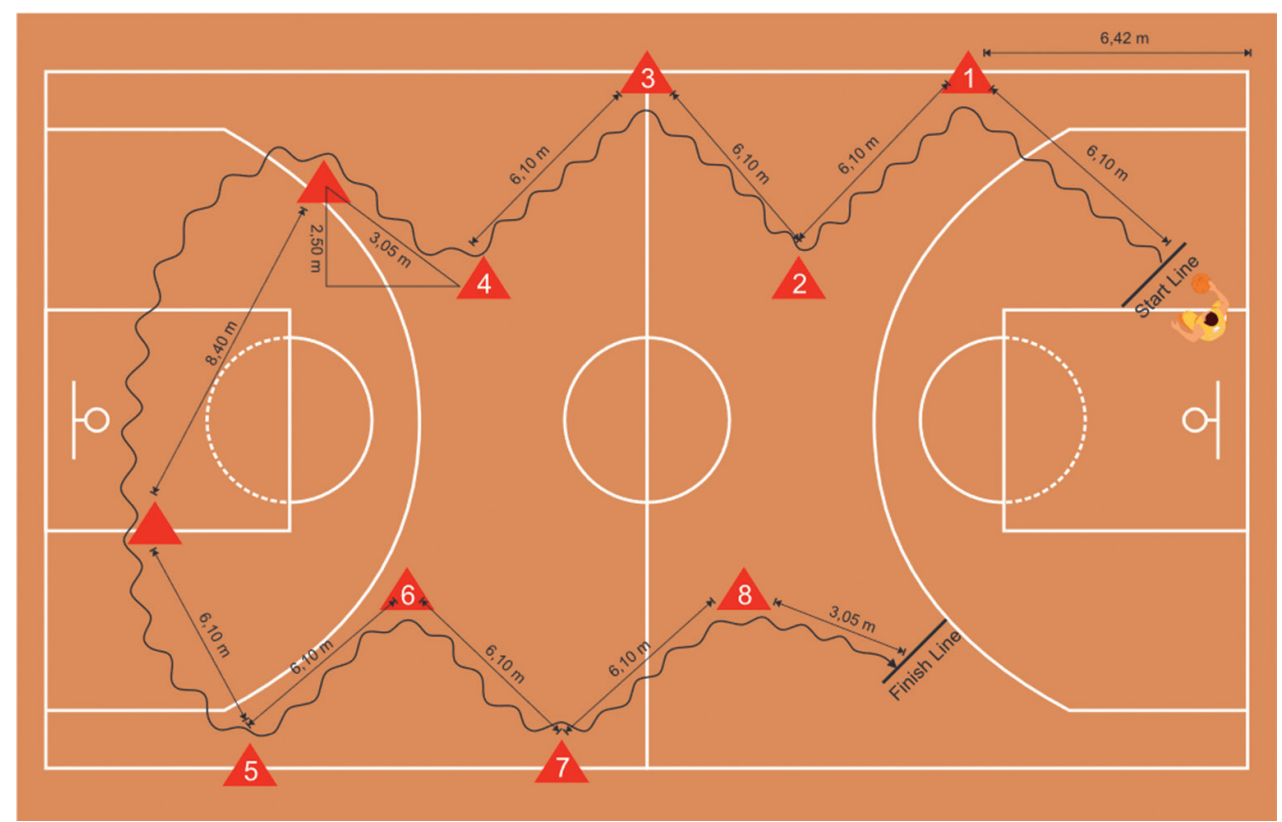

Figure 1. The illustration of the dribbling protocol. 
nize both the kinetic and kinematic systems, the players were asked to lift their either left or right leg three times off the ground just before starting dribbling. That was the signal for the alignment of time-lapse of both systems.

The dribbling testing protocol is presented in Figure 1. The players dribbled the ball in full speed down the dribbling route (from the start line until the finish line) twice: first in the non-fatigued and then in the fatigued state. Every participant started his task execution with his right hand and changed movement directions when they approached the cones (from cone 1 to cone 8). The skill of the front COD in dribbling was performed at cones $1,2,5$, and 6 , and the spin move was performed at cones $3,4,7$, and 8 . Distance between the middle of the start line and the court baseline was 2.10 meters and distance between each two cones, where the players changed direction, was 6.10 meters. The photocells were placed at the start and finish line for measuring dribbling speed.

After the first dribbling test (non-fatigued state), the players were asked to perform the fatigue protocol: a 300 -meter shuttle run $(15 \times 20 \mathrm{~m}$ with COD of $180^{\circ}$ ). This fatigue protocol was employed due to having similarities with actual game situations in which a player runs forward and backward consecutively and its reliability was previously verified (Callister, et al., 2010; Sporiš, et al., 2014). During the 300-meter shuttle run, the players were instructed to sprint as fast as possible and the sprint time was recorded by photocells (WittyGate, Microgate, Bolzano, Italy). Afterwards, the players performed the dribbling task once again so that we can observe the difference in dribbling kinematic and kinetic COD performance between the nonfatigued and fatigued states.

\section{Variables}

Analysis of kinematic and kinetic variables was conducted on two types of dribbling: front COD and spin move. These two types of COD have been previously identified as the basic dribbling skills (Krause \& Nelson, 2018). The following variables were measured:

The dribbling time the players needed to complete the testing protocol (in seconds).

For the front COD: the lowest pelvis position $\left(\mathrm{PP}_{\min }\right)(\mathrm{cm})$, the highest pelvis position $\left(\mathrm{PP}_{\max }\right)(\mathrm{cm})$, and the average pelvis position $\left(\mathrm{PP}_{\text {aver }}\right)(\mathrm{cm})$ at the moment when the players performed the front COD; the minimum angle in the knee joint of the outside leg $\left(\mathrm{KA}_{\min }\right)\left(^{\circ}\right)$; the maximum angular velocity in the knee joint of the outside leg during the concentric phase $\left(\right.$ Knee_ $\left.A V_{\max }\right)(\%)$; the maximum angular velocity in the wrist joint (Wrist $\left.A V_{\text {max }}\right)(\%$ ) from the point at which the player flexed his outside hand to switch the ball to his inside hand; the first step velocity $(\mathrm{FSV})(\mathrm{cm})$ at the moment the player started to move to the reverse direction (COD has been performed); the maximum force of the outside foot during the concentric phase $\left(\right.$ Force $\left._{\max }\right)(\mathrm{N})$.

In Figure 2 the movement pattern of the front COD in dribbling is presented.

For the spin move: the lowest pelvis position $\left(\mathrm{PP}_{\text {min }}\right)(\mathrm{cm})$, the highest pelvis position $\left(\mathrm{PP}_{\max }\right)(\mathrm{cm})$, and the average pelvis position $\left(\mathrm{PP}_{\text {aver }}\right)(\mathrm{cm})$ during the spin move performance; the pelvis maximum velocity during the spin move executed by the rota-

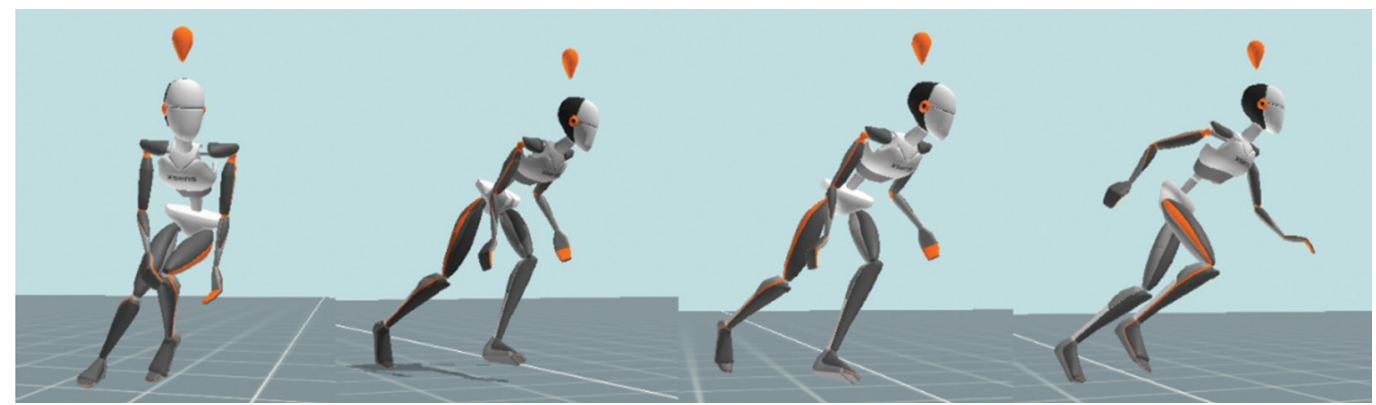

Figure 2. The movement pattern of the front change of direction.

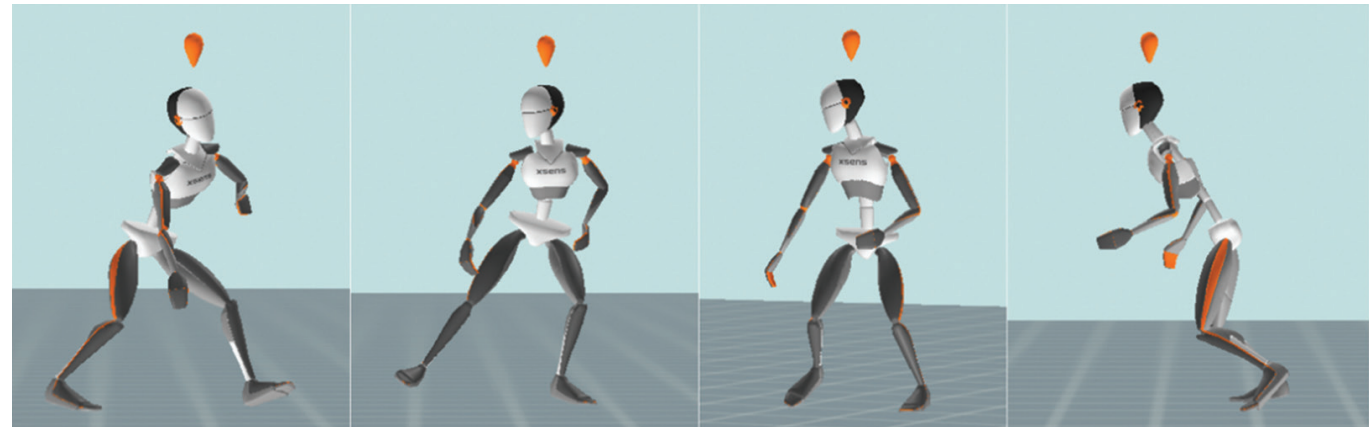

Figure 3. The movement pattern of the spin move. 
tion of the pelvis $\left(\mathrm{PV}_{\max }\right)(\mathrm{m} / \mathrm{s})$; the minimum angle in the knee joint of the inside leg $\left(\mathrm{KA}_{\min }\right)\left(^{\circ}\right)$; the maximum angular velocity in the knee joint of the inside leg during the concentric phase (Knee $\left.\mathrm{AV}_{\text {max }}\right)(\%)$; velocity of the first step (FSV) (m/s); the maximum force of the inside foot during the concentric phase $\left(\right.$ Force $\left._{\max }\right)(\mathrm{N})$.

Figure 3 demonstrate the movement pattern of the spin move.

\section{Statistical analysis}

With the use of the $\mathrm{G}^{*}$ power program, the sample size (the number) of dribbles with COD needed for inferential statistical analysis was calculated $(n=98)$ at statistical significance of $p<.05$; statistical power 0.8 ; effect size 0.3 , and two groups. Kinematic and kinetic parameters of dribbling were measured in every COD performance during a dribbling execution (giving a total of 224 CODs performed, 56 of each type in each state of fatigue). Unfortunately, 24 CODs were excluded from the final analysis due to some technical issues with the equipment or movement pattern execution. Overall, a total of 200 properly executed and measured CODs (50 of each type in each state of fatigue) was analyzed in this study.

All analyses were executed in the statistical package Statistica, version 13.5.0.17 (TIBCO Software Inc, Palo Alto, CA, USA; release date: November 2018). Values were expressed as mean \pm standard deviation. Basic descriptive parameters were calculated for all the measured variables. The normality of the data distribution was confirmed by a Shapiro-Wilk test. To verify the differences in the kinematic variables between the fatigued and nonfatigued states, an analysis of variance (ANOVA) for repeated measurements was applied. To determine the difference in dribbling speed between the non-fatigued and fatigued states, a $t$-test for dependent samples was conducted and the effect size was determined using the Cohen's d. The level of statistical significance was set at $\alpha=.05$.

\section{Results}

\section{Front COD}

Table 1 shows that there was a significant difference between the fatigued and non-fatigued state $(\mathrm{p}=0.000)$.

Table 1. The results of ANOVA for repeated measurements (for groups)

\begin{tabular}{lccc}
\hline Test & Value & $\mathbf{F}$ & $\mathbf{p}$ \\
\hline Wilks & 0.75 & 3.87 & $0.000^{*}$ \\
\hline
\end{tabular}

Note. * Marked values were significant when $p<.05$.

Table 2 and Figure 4 provide the descriptive parameters and results of ANOVA for repeated kinematic and kinetic measurements executed in the fatigued and non-fatigued states. First, the mean value of $\mathrm{KNEE} \_\mathrm{AV}_{\max }$ significantly decreased in the

Table 2. Descriptive parameters and results of ANOVA for repeated measurements of the fatigued and non-fatigued states

\begin{tabular}{|c|c|c|c|c|c|c|c|c|}
\hline Variable & Group & $\mathbf{N}$ & Mean & Min & Max & SD & $\mathbf{F}$ & p \\
\hline \multirow{2}{*}{$\mathrm{PP}_{\min }(\mathrm{cm})$} & Non-fatigued & 50 & 76.79 & 64.93 & 91.30 & 6.47 & \multirow{2}{*}{0.15} & \multirow{2}{*}{0.700} \\
\hline & Fatigued & 50 & 77.31 & 65.56 & 93.80 & 6.92 & & \\
\hline \multirow{2}{*}{$\mathrm{PP}_{\max }(\mathrm{cm})$} & Non-fatigued & 50 & 90.51 & 76.12 & 107.05 & 6.53 & \multirow{2}{*}{0.06} & \multirow{2}{*}{0.804} \\
\hline & Fatigued & 50 & 90.85 & 75.03 & 108.93 & 6.96 & & \\
\hline \multirow{2}{*}{$\mathrm{PP}_{\text {aver }}(\mathrm{cm})$} & Non-fatigued & 50 & 82.72 & 71.21 & 98.16 & 6.08 & \multirow{2}{*}{0.29} & \multirow{2}{*}{0.589} \\
\hline & Fatigued & 50 & 83.42 & 72.25 & 99.98 & 6.86 & & \\
\hline \multirow{2}{*}{$\mathrm{KA}_{\text {min }}\left({ }^{\circ}\right)$} & Non-fatigued & 50 & 118.70 & 103.52 & 132.56 & 7.16 & \multirow{2}{*}{0.14} & \multirow{2}{*}{0.707} \\
\hline & Fatigued & 50 & 119.23 & 102.58 & 132.88 & 6.93 & & \\
\hline \multirow{2}{*}{ KNEE_AV $\max (\% / s)$} & Non-fatigued & 50 & 429.72 & 222.59 & 664.81 & 109.01 & \multirow{2}{*}{4.97} & \multirow{2}{*}{$0.028^{*}$} \\
\hline & Fatigued & 50 & 378.63 & 180.72 & 661.01 & 119.79 & & \\
\hline \multirow{2}{*}{ WRIST_AV ${ }_{\max }\left({ }^{\circ} / \mathbf{s}\right)$} & Non-fatigued & 50 & 387.56 & 115.26 & 709.03 & 164.31 & \multirow{2}{*}{7.62} & \multirow{2}{*}{$0.007^{*}$} \\
\hline & Fatigued & 50 & 300.85 & 96.52 & 771.62 & 149.34 & & \\
\hline \multirow{2}{*}{$\mathrm{FSV}(\mathrm{m} / \mathrm{s})$} & Non-fatigued & 50 & 4.78 & 2.58 & 7.32 & 1.06 & \multirow{2}{*}{1.09} & \multirow{2}{*}{0.299} \\
\hline & Fatigued & 50 & 4.55 & 1.26 & 6.21 & 1.14 & & \\
\hline \multirow{2}{*}{ Force $_{\max }(\mathrm{N})$} & Non-fatigued & 50 & 1782.26 & 1109.16 & 2747.52 & 347.06 & \multirow{2}{*}{8.89} & \multirow{2}{*}{$0.004^{*}$} \\
\hline & Fatigued & 50 & 1608.42 & 1132.95 & 2043.40 & 222.52 & & \\
\hline
\end{tabular}

Note. ${ }^{*} \mathrm{p}<.05 ; \mathrm{PP}_{\min }-$ the lowest pelvis position at the moment when the players performed the front change; $\mathrm{PP}_{\max }-\mathrm{the}_{\mathrm{mighest}}$ pelvis position at the moment when the players performed the front change; $\mathrm{PP}_{\text {aver }}-$ the average pelvis position at the moment when the players performed the front change; $\mathrm{KA}_{\min }$ - the minimum angle in the knee joint of the outside leg; $\mathrm{Knee}_{-} \mathrm{AV}_{\text {max }}-\mathrm{the}_{\text {maximum }}$ angular velocity in the knee joint of the outside leg during the concentric phase; Wrist_AV $\mathrm{V}_{\text {max }}-$ the maximum angular velocity in the wrist joint from the point that the players flexed their outside hand to switch the ball to the inside hand; FSV - the first step velocity at the moment when the players performed the front change; Force ${ }_{\max }-$ the maximum force of the outside foot during the concentric phase. 

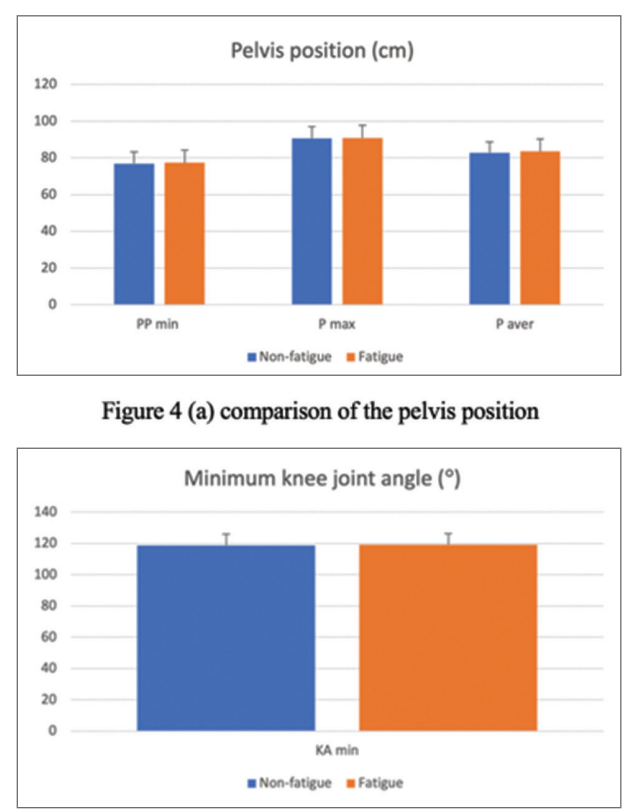

Figure 4 (c) comparison of the minimum knee angle

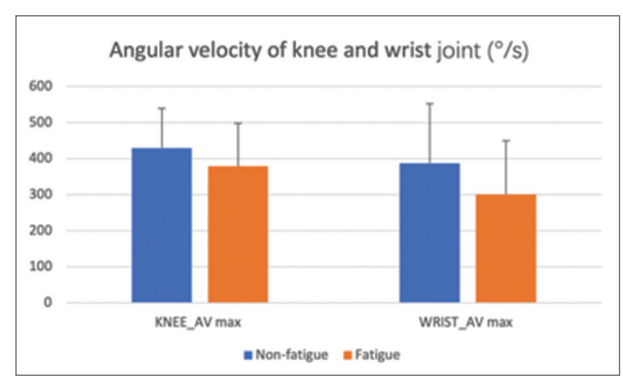

Figure 4 (b) comparison of the angular velocity

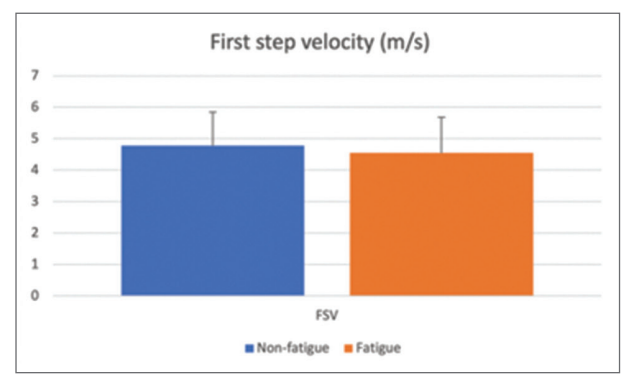

Figure 4 (d) comparison of the first step velocity

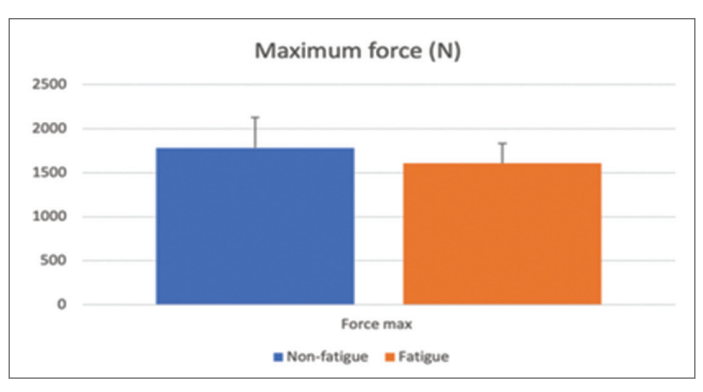

Figure 4 (e) comparison of the maximum force

Figure 4. The comparison of the variables for the front COD.

fatigued compared to the non-fatigued state (fatigued $=378.63 \%$; non-fatigued $=429.72 \%$ s; $p=.028$ ). Additionally, the mean value of WRIST_AV $\mathrm{AV}_{\max }$ considerably decreased in the fatigued compared to the non-fatigued state (fatigued $=300.85 \%$ s; nonfatigued $=387.56 \%$; $p=.007)$. Furthermore, the mean value of Force max $_{\text {ax }}$ dramatically decreased in the fatigued compared to the non-fatigued state (fatigued $=1608.42 \mathrm{~N}$; non-fatigued=1782.26 N; $\mathrm{p}=.004)$. Second, the mean value of FSV was lower in the fatigued than in the non-fatigued state (fatigued $=4.55 \mathrm{~m} / \mathrm{s}$; non-fatigued $=4.78 \mathrm{~m} / \mathrm{s}$ ) but there was no significant difference between the two variables $(\mathrm{p}=.229)$. Last, the $\mathrm{PP}_{\min }, \mathrm{PP}_{\max }$ and $\mathrm{PP}_{\text {aver }}$ all slightly increased in the fatigued state compared to the non-fatigued state. Likewise, the $\mathrm{KA}_{\min }$ was higher in the fatigued state than in the non-fatigued state (fatigued $=119.23^{\circ}$; non-fatigued $=118.70^{\circ}$ ). However, there was no significant difference in terms of $\mathrm{PP}_{\min }(\mathrm{p}=.700), \mathrm{PP}_{\max }(\mathrm{p}=.804), \mathrm{PP}_{\text {aver }}$ $(\mathrm{p}=.589)$ and $\mathrm{KA}_{\min }(\mathrm{p}=.707)$.

\section{Spin move}

The analysis of variance (ANOVA) for repeated measurements was used to observe the biomecha- nical difference in dribbling performance between the non-fatigued and fatigued states. As Table 3 shows, there was a significant difference between the two groups of variables $(\mathrm{p}=.003)$.

Table 3. The results of ANOVA for repeated measurements (for groups)

\begin{tabular}{lccc}
\hline Test & Value & F & $\boldsymbol{p}$ \\
\hline Wilks & 0.78 & 3.20 & $0.003^{*}$ \\
\hline
\end{tabular}

Note. * Marked values were significant when $p<.05$.

Table 4 and Figure 5 present the descriptive parameters and results of ANOVA for repeated kinematic and kinetic measurements executed in the fatigued and non-fatigued states. First, the mean value of $\mathrm{PV}_{\max }$ significantly decreased in the fatigued state compared to the non-fatigued state (fatigued $=2.79 \mathrm{~m} / \mathrm{s}$; non-fatigued $=3.15 \mathrm{~m} / \mathrm{s} ; \mathrm{p}=.000$ ). Likewise, the mean value of KNEE_AV $\mathrm{Amax}_{\max }$ was dramatically decreased in the fatigued state (fatigued $=286.90 \%$; non-fatigued $=328.63 \%$; $p=.020$ ). Moreover, FSV was considerably lower in the fatigued than in the non-fatigued state (fatigued $=7.41$ $\mathrm{m} / \mathrm{s}$; non-fatigued $=7.76 \mathrm{~m} / \mathrm{s} ; \mathrm{p}=.010$ ). Second, the 
Table 4. Descriptive parameters and results of ANOVA for repeated measurements of the fatigued and non-fatigued states

\begin{tabular}{|c|c|c|c|c|c|c|c|c|}
\hline Variable & Group & $\mathbf{N}$ & Mean & Min & Max & SD & $\mathbf{F}$ & p \\
\hline \multirow{2}{*}{$\mathrm{PP}_{\min }(\mathrm{cm})$} & Non-fatigued & 50 & 78.82 & 70.16 & 98.25 & 6.59 & \multirow{2}{*}{0.76} & \multirow{2}{*}{0.386} \\
\hline & Fatigued & 50 & 80.02 & 69.23 & 96.670 & 7.25 & & \\
\hline \multirow{2}{*}{$\mathrm{PP}_{\max }(\mathrm{cm})$} & Non-fatigued & 50 & 92.08 & 78.57 & 109.95 & 7.39 & \multirow{2}{*}{0.46} & \multirow{2}{*}{0.498} \\
\hline & Fatigued & 50 & 93.07 & 80.75 & 106.43 & 7.20 & & \\
\hline \multirow{2}{*}{$\mathrm{PP}_{\text {aver }}(\mathrm{cm})$} & Non-fatigued & 50 & 86.71 & 76.35 & 104.56 & 7.19 & \multirow{2}{*}{0.20} & \multirow{2}{*}{0.656} \\
\hline & Fatigued & 50 & 87.36 & 74.93 & 102.00 & 7.42 & & \\
\hline \multirow{2}{*}{$\mathrm{PV}_{\max }(\mathrm{m} / \mathrm{s})$} & Non-fatigued & 50 & 3.15 & 2.42 & 4.09 & 0.37 & \multirow{2}{*}{16.88} & \multirow{2}{*}{$0.000^{*}$} \\
\hline & Fatigued & 50 & 2.79 & 1.12 & 3.69 & 0.49 & & \\
\hline \multirow{2}{*}{$\mathrm{KA}_{\min }\left({ }^{\circ}\right)$} & Non-fatigued & 50 & 111.98 & 92.85 & 135.21 & 9.61 & \multirow{2}{*}{1.14} & \multirow{2}{*}{0.288} \\
\hline & Fatigued & 50 & 114.21 & 91.92 & 141.67 & 11.28 & & \\
\hline \multirow{2}{*}{ KNEE_AV $V_{\max }\left({ }^{\circ} / \mathbf{s}\right)$} & Non-fatigued & 50 & 328.63 & 202.29 & 563.13 & 90.92 & \multirow{2}{*}{5.60} & \multirow{2}{*}{$0.020^{*}$} \\
\hline & Fatigued & 50 & 286.90 & 49.10 & 559.78 & 85.33 & & \\
\hline \multirow{2}{*}{$\mathrm{FSV}(\mathrm{m} / \mathrm{s})$} & Non-fatigued & 50 & 7.76 & 6.43 & 9.07 & 0.62 & \multirow{2}{*}{6.83} & \multirow{2}{*}{$0.010^{*}$} \\
\hline & Fatigued & 50 & 7.41 & 4.87 & 8.84 & 0.71 & & \\
\hline \multirow{2}{*}{ Force $_{\max }(\mathrm{N})$} & Non-fatigued & 50 & 1736.77 & 963.54 & 2786.40 & 415.88 & \multirow{2}{*}{3.64} & \multirow{2}{*}{0.059} \\
\hline & Fatigued & 50 & 1583.00 & 982.80 & 2838.60 & 389.24 & & \\
\hline
\end{tabular}

Note. ${ }^{*} \mathrm{p}<.05 ; \mathrm{PP}_{\min }$ - the lowest pelvis position at the moment when the players performed the spin move; $\mathrm{PP}_{\max }-$ the highest pelvis position at the moment when the players performed the spin move; $\mathrm{PP}_{\text {aver }}-$ the average pelvis position at the moment when the players performed the spin move; $\mathrm{PV}_{\max }$ - the maximum velocity of the pelvis at the moment when the players performed the spin move by rotating their pelvis; $\mathrm{KA}_{\min }$ - the minimum angle in the knee joint of the inside leg; $K$ nee_A $\mathrm{V}_{\max }$ - the maximum angular velocity in the knee joint of the inside leg during the concentric phase; FSV - the first step velocity at the moment when the players performed the spin move; Force $_{\max }$ - the maximum force of the inside foot during the concentric phase.

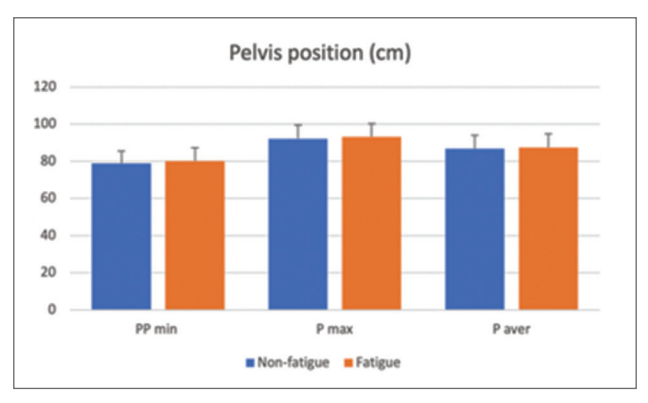

Figure 5 (a) comparison of the pelvis position

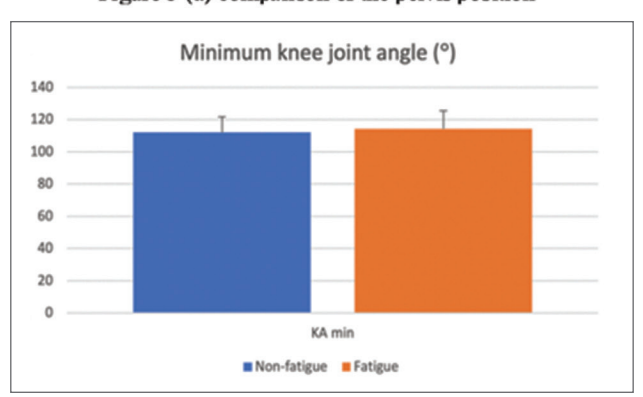

Figure 5 (c) comparison of the knee angle

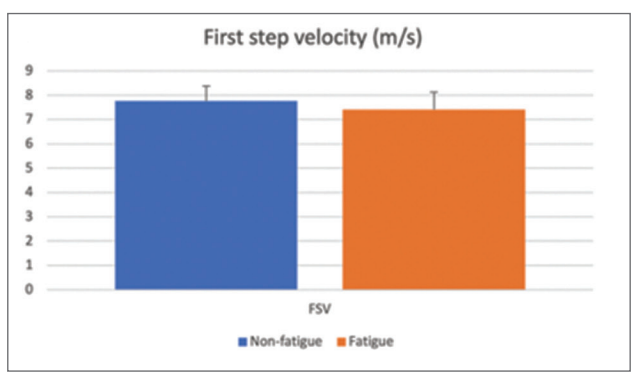

Figure 5 (e) comparison of the first step velocity

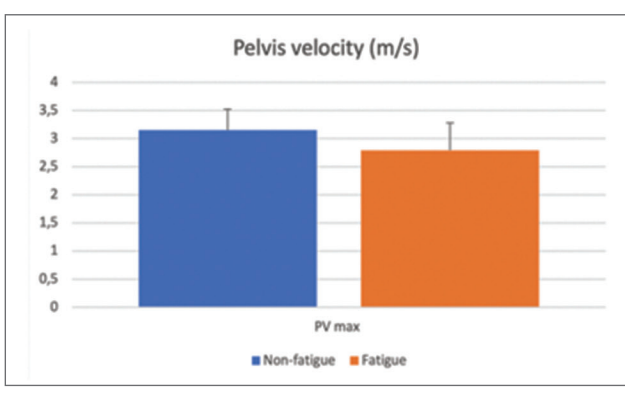

Figure 5 (b) comparison of the pelvis velocity

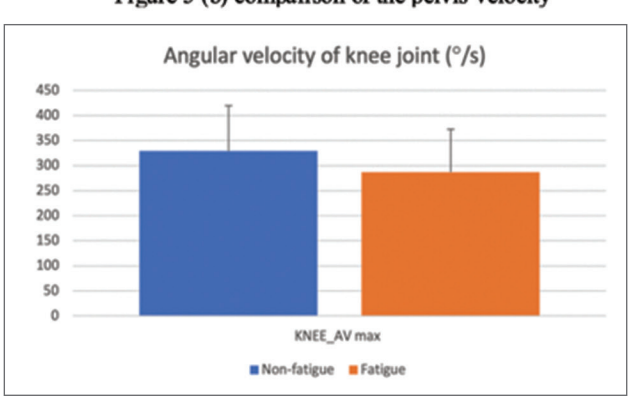

Figure 5 (d) comparison of the angular velocity

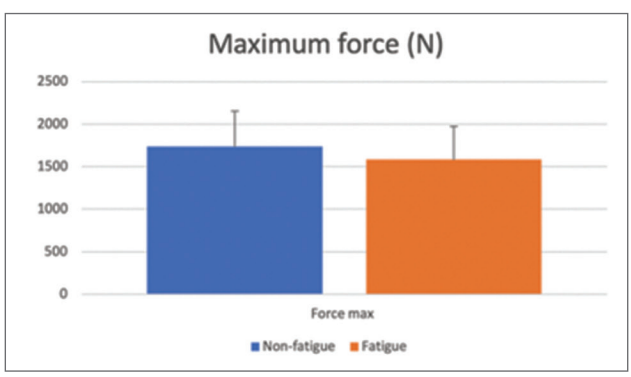

Figure 5 (f) comparison of the maximum force

Figure 5. The comparison of the variables for the spin move. 
Table 5. The results in dribbling speed of the t-test for dependent samples

\begin{tabular}{lccccc}
\hline Group & N & Mean $(\mathbf{s})$ & SD & p & Cohen's (d) \\
\hline Non-fatigued & 14 & 17.50 & 0.87 & \multirow{2}{*}{$0.002^{*}$} & 1.001 \\
\hline Fatigued & 14 & 18.53 & 1.52 & & \\
\hline
\end{tabular}

Note. ${ }^{*}$ Marked values were significant when $p<.05$.

Table 6. Descriptive statistics of heart rate (HR) and blood lactate (BL) variables between the non-fatigued and fatigued states

\begin{tabular}{llcccc}
\hline Variable & N & Mean & Minimum & Maximum & SD \\
\hline HR_B_WU (beats/min) & 14 & 79.07 & 58.00 & 102.00 & 11.47 \\
\hline HR_A_FT (beats/min) & 14 & 173.36 & 156.00 & 194.00 & 11.84 \\
\hline HR_A_FP (beats/min) & 14 & 188.57 & 173.00 & 203.00 & 9.39 \\
\hline HR_D_ST max $_{\text {(beats/min) }}$ & 14 & 178.86 & 164.00 & 197.00 & 10.28 \\
\hline BL_B_WU (mmol/l) & 14 & 1.26 & 0.60 & 1.70 & 0.35 \\
\hline BL_A_WU (mmol/l) & 14 & 2.74 & 0.70 & 5.80 & 1.55 \\
\hline BL_A_FT (mmol/l) & 14 & 5.55 & 2.60 & 10.00 & 2.52 \\
\hline BL_A_FP (mmol/l) & 14 & 11.06 & 6.70 & 15.70 & 3.19 \\
\hline
\end{tabular}

Note. BL_B_WU - the players' heart rate before warm-up; HR_A_F - the players' heart rate after the first testing (non-fatigued state); HR_A_FP - the players' heart rate after the fatigue protocol; HR_D_ST max - the players' heart rate during second testing (fatigued

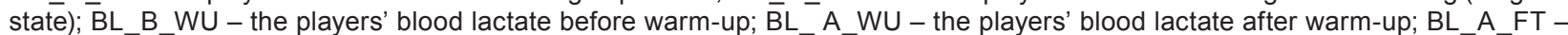
the players' blood lactate after the first testing (non-fatigued state); BL_A_FP - the players' blood lactate after the fatigue protocol (fatigued state).

Force $_{\text {max }}$ in the fatigued state decreased compared to the non-fatigued state but no statistical significance was reached (fatigued $=1583 \mathrm{~N}$; non-fatigued $=1736.77 \mathrm{~N} ; \mathrm{p}=.059)$. Last, similar to the results for the front COD dribbling, the mean values of $\mathrm{PP}_{\min }, \mathrm{PP}_{\max }, \mathrm{PP}_{\text {aver }}$ and $\mathrm{KA}_{\min }$ were slightly higher in the fatigued state than in the non-fatigued state but no significant differences were obtained $-\mathrm{PP}_{\text {min }}$ ( $\mathrm{p}=.386), \mathrm{PP}_{\max }(\mathrm{p}=.498), \mathrm{PP}_{\text {aver }}(\mathrm{p}=.656)$, and $\mathrm{KA}_{\min }$ $(\mathrm{p}=.288)$.

To determine the difference in dribbling speed between the non-fatigued and fatigued states, a $t$-test for dependent samples was applied. As can be seen from Table 5, the players needed significantly $(p=.002)$ more time in the fatigued state compared to the non-fatigued state (fatigued $=18.53 \mathrm{~s}$; non-fatigued $=17.50 \mathrm{~s}$ ).

\section{The physiological response during testing}

Table 6 shows an overview of the participants' heart rate and blood lactate values in the non-fatigued and fatigued states. The mean HR value was 79.07 beats/min and 188.57 beats/min before warm-up (non-fatigued state) and after the fatigue protocol (fatigued state), respectively. Additionally, the mean $\mathrm{BL}$ value was $1.26 \mathrm{mmol} / \mathrm{L}$ and 11.06 $\mathrm{mmol} / \mathrm{L}$ before warm-up (non-fatigued state) and after the fatigue protocol (fatigued state), respectively. The aforementioned results revealed that there was a significant difference between the non-fatigued and fatigued states in fatigue levels.

\section{Discussion and conclusions}

Dribbling with COD is motorically the most complex type of dribbling and players perform it frequently during competition due to a high level of pressure from defenders (Krause \& Nelson, 2018). Previous studies have reported that fatigue has a negative influence on basketball players' performance (Erculj \& Supej, 2009; Mulazimoglu, et al., 2017). Surprisingly, little is known about the effect of fatigue on kinematics and kinetics of basketball dribbling. The aim of this study was to determine the effect of fatigue on kinematics and kinetics of dribbling with COD as well as the effect of fatigue on dribbling speed. The results of the present study showed that there was a significant difference in dribbling kinematics and kinetics between the fatigued and non-fatigued states. Additionally, dribbling speed significantly decreased in the fatigued state compared to the non-fatigued state. The findings are in line with our previously formulated hypotheses.

In this study, the mean value of the players' HR was $79.07 \pm 11.47$ beats $/ \mathrm{min}$ in the state before warm-up (non-fatigued state) and $188.57 \pm 9.39$ beats/ $\mathrm{min}$ in the state after the fatigue protocol (fatigued state). Additionally, the mean value of the players' BL concentration was $1.26 \pm 0.35 \mathrm{mmol} / \mathrm{L}$ in the state before warm-up (non-fatigued state) and 11.06 \pm 3.19 $\mathrm{mmol} / \mathrm{L}$ in the state after the fatigue protocol (fatigued state). There were some studies investigating the response of $\mathrm{HR}$ and $\mathrm{BL}$ to the real game situation (McInnes, Carlson, Jones, \& McKenna, 1995; 
Torres-Ronda, Ric, Llabres-Torres, de Las Heras, \& Schelling, 2016). Torres-Ronda et al. (2016) assessed players' HR during a friendly basketball game, reporting that the peak HR was 198 beats/min. Another study by McInnes et al. (1995) observed the physiological response to basketball competition, stating that the mean maximum BL concentration for all subjects was $8.5 \pm 3.1 \mathrm{mmol} / \mathrm{L}$, with the highest individual having $13.2 \mathrm{mmol} / \mathrm{L}$. Given the aforementioned results, it can be concluded in this study that the conditions during testing in the fatigued state were similar to real basketball competition (game speed).

For the front change (FCOD) and spin move (SM), the selected variables of this study were pelvis position, $\mathrm{KA}_{\min }, \mathrm{KNEE} \mathrm{AV}_{\max }, \mathrm{WRIST}$

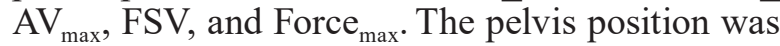
used to observe the difference of the center of mass between the non-fatigued and fatigued states. Similarly, the $\mathrm{KA}_{\min }$ was used to observe the lowest position of the knee so to identify if the players lowered their body when the defenders (cones) were close to the ball handler. The assumption was that, if the aforementioned variables in the fatigued state had higher values, it would mean that the player, his center of mass, was in a higher position, which might consequently induce the possibility of losing ball possession and affect the realization of spatial and temporal advantage over the defender. Addi-

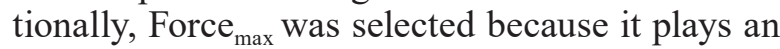
important role in the players' FSV. Namely, FSV determines if a player can pass by the defender successfully. Furthermore, the KNEE_AV max $_{\text {mas }}$ selected because the players with a higher KNEE $\mathrm{AV}_{\max }$ could generally perform a quick first step. Moreover, the players with higher WRIST_AV $\mathrm{AV}_{\max }$ are able to switch the ball from the outside to the inside hand quickly so that they cannot only protect the ball well but also make a quick COD to pass by the defenders. Last, the $\mathrm{PV}_{\max }$ in $\mathrm{SM}$ was selected because a higher velocity of pelvis rotation was not only critical for ball possession keeping but it also facilitated achieving a spatial and temporal advantage over the defender.

Consistent with literature, this study found that there were significant differences between the non-fatigued and fatigued states in the kinematics of FCOD and SM, which was in agreement with previous studies showing that the kinematic parameters of basketball skills changed when the players were under the influence of fatigue (Erculj \& Supej, 2006, 2009; Rupčić, et al., 2020). Additionally, the current study found that there was a statistically significant difference in Force ${ }_{\text {max }}$ (kinetic) with respect to FCOD between the fatigued and non-fatigued states (fatigued $=1608.42 \mathrm{~N}$; non-fatigued $=1782.26 \mathrm{~N} ; \mathrm{p}=.004$ ). This finding is in line with previous studies confirming that fatigue can cause the reduction in the capacity of muscles to generate force (Arora, Budden, Byrne, \& Behm, 2015; Morin, Samozino, Edouard, \& Tomazin, 2011; Wan, Qin, Wang, Sun, \& Liu, 2017), which results in a player who is unable to continue moving at the same level of performance (Cortes, Onate, \& Morrison, 2014; Morin, et al., 2011). However, the Force $_{\text {max }}$ did not significantly decrease in the fatigued state in terms of SM (fatigued $=1583 \mathrm{~N}$; nonfatigued $=1736.77 \mathrm{~N} ; \mathrm{p}=.059)$. A possible explanation for this result may be the technical difference between the FCOD and SM execution. When players perform SM, they first make a forceful step with the inside foot, then the pressure on the inside foot is shifted onto the outside foot with the rotation of the pelvis following the first step (Krause $\&$ Nelson, 2018). In terms of FCOD, however, the player conducts the first step by fully pressing the ground with the same foot. Therefore, the foot pressure of the support leg during the concentric phase is supposed to be lower in SM compared to FCOD. As a result, the Force max $_{\text {max }}$ did not significantly decrease in the fatigued state.

The results of this study revealed that dribbling speed significantly decreased in the fatigued state $(p=.002)$, corresponding to previous studies which concluded that fatigue had a negative influence on basketball players' performance (Erculj \& Supej, 2009; Mulazimoglu, et al., 2017; Thorpe, et al., 2017). These results may be explained by the fact that the reduction of force production leads to the decrease of dribbling speed as it has been proved that there is a strong correlation between velocity and force (Janicijevic, et al., 2020; Zivkovic, Djuric, Cuk, Suzovic, \& Jaric, 2017). Furthermore, previous studies have pointed out that a greater lower body strength (Spiteri, Cochrane, Hart, Haff, $\&$ Nimphius, 2013) and the subsequent application of a greater force and impulse (Spiteri, et al., 2014) enable athletes to perform a more effective and rapid motor response. In accordance with the present results, numerous studies have found the same result that sprint speed decreases when the subjects are in the fatigued state (Dal Pupo, Detanico, AcheDias, \& Santos, 2017; Morin, et al., 2011). Morin et al. (2011) investigated the effect of fatigue on force production and force application technique during repeated sprints. Sprint speed and force production dramatically decreased. Furthermore, Dal Pupo et al. (2017) studied the fatigue effect of a simulated futsal match protocol on sprint performance and the kinematics of the lower limbs, demonstrating a significant decrement in sprint speed.

In our study, the KNEE_AV $\mathrm{AV}_{\max }(\mathrm{p}=.028)$ and WRIST_AV $\mathrm{Amax}_{\max }(\mathrm{p}=.007)$ in the fatigued state significantly decreased compared to the non-fatigued state with respect to FCOD. Additionally, the mean value of KNEE_AV $\mathrm{AV}_{\max }$ dramatically decreased in the fatigued state $(p=.020)$ regarding SM. These results may be explained by the fact that fatigue 
caused the reduction in muscle strength, velocity, and coordination (Janicijevic, et al., 2020; Morin, et al., 2011; Zivkovic, et al., 2017), which ultimately reduced angular velocities in individual joint systems. Our study can explain the decrease of the FSV in both FCOD and SM by the fact that fatigue caused the decrease in force production and then further led to the deterioration of FSV. Similarly, the mean value of $\mathrm{PV}_{\max }$ in $\mathrm{SM}$ significantly decreased in the fatigued state $(\mathrm{p}=.000)$, which was likely to be associated with this factor.

In this study, the pelvis position $\left(\mathrm{PP}_{\min }, \mathrm{PP}_{\max }\right.$, $\mathrm{PP}_{\text {aver }}$ ) was higher and $\mathrm{KA}_{\min }$ was larger in the fatigued state than in the non-fatigued state regardless of whether in FCOD or SM. Previous studies have stated that decreases in the lower limb muscle activation due to fatigue could result in changes in pelvis position, and the reduction in strength tended to increase the players' center of mass (Lessi, dos Santos, Batista, de Oliveira, \& Serrão, 2017; Lessi $\&$ Serrão, 2017). As a result, theoretically, the $\mathrm{KA}_{\text {min }}$ is supposed to also be increased when the players' pelvis position increases.

In summary, the major conclusion drawn from this study according to the results was that fatigue significantly affected the kinematics and kinetics of basketball dribbling. Additionally, dribbling speed significantly decreased when the players were under the fatigued state. The higher pelvis position, the lower angular velocity in the knee and wrist joint, and the lower force production when the players are under the fatigued state may induce their inability to take advantage over the defender successfully. Additionally, the decrease in dribbling speed under the fatigued state will make players less able to pass quickly by the defender during the fast break and transition to offense, which consequently makes them lose the opportunity of scoring.

\section{Practical application}

The findings of this study have provided evidence for the coaching staff why they are required to design appropriate training programs to optimize the players' fatigue resistance needed in dribbling. There is an exceptional need, after the skill has been adopted, to practice it under the conditions of high load and game speed in order to decrease the expected reduction in execution efficiency. Segments that have shown statistical significance should be in the focus of expert coaches being able to evaluate skill performance and constantly improve it using a large number of training operators under high load. It is important to ultimately achieve skill performance automatism in players whose skill execution will remain close to perfect even under the conditions of extreme load in order to be able to respond to the challenges of modern basketball in which defensive pressure on the player with the ball is extremely high. Only players who can demonstrate a high level of skill performance under high loads (both cognitive and physical) can find the best solution in spatial and temporal on-court alignment and ultimately achieve exceptional situation-related efficiency.

\section{References}

Andrić, M. (2011). Analysis of frequency and efficiency of using dribble in the European basketball championship in Poland in 2009. Fizička kultura, 65(1), 52-59.

Arias, J.L., Argudo, F.M., \& Alonso, J.I. (2012). Effect of ball mass on dribble, pass, and pass reception in 9-11-year-old boys' basketball. Research Quarterly in Exercise and Sport, 83(3), 407-412. doi: 10.1080/02701367.2012.10599875

Arias-Estero, J.L. (2013). Opportunities for and success in dribbling, passing, receiving, and shooting in youth basketball. International Journal of Sports Science Coaching, 8(4), 703-711. doi: 10.1260/1747-9541.8.4.703

Arora, S., Budden, S., Byrne, J.M., \& Behm, D.G. (2015). Effect of unilateral knee extensor fatigue on force and balance of the contralateral limb. European Journal of Applied Physiology, 115(10), 2177-2187. doi: 10.1007/ s00421-015-3198-5

Balsalobre-Fernández, C., Bishop, C., Beltrán-Garrido, J.V., Cecilia-Gallego, P., Cuenca-Amigó, A., Romero-Rodríguez, D., \& Madruga-Parera, M. (2019). The validity and reliability of a novel app for the measurement of change of direction performance. Journal of Sports Sciences, 37, 2420-2424. doi: 10.1080/02640414.2019.1640029

Belić, M., Radin, L., Brkljača Bottegaro, N., Beer, L., Blanka, B.M., Stanin, D., \& Vrbanac, Z. (2016). Reliability of lactate scout portable analyzer in agility dogs during multiple measurements. Acta Veterinaria-Beograd, 66, 549-555.

Calleja-González, J., Terrados, N., Mielgo-Ayuso, J., Delextrat, A., Jukic, I., Vaquera, A., Torres, L., Schelling, X., Stojanovic, M., \& Ostojic, S.M. (2016). Evidence-based post-exercise recovery strategies in basketball. Phys Sportsmed, 44, 74-78. doi: 10.1080/00913847.2016.1102033

Callister, R., Miller, A., Aguiar, E., Dascombe, B., Smith, C., Clark, L., \& Rogers, T. (2010). Blood lactate levels support classification of the $300 \mathrm{~m}$ shuttle run as an anaerobic capacity field test. Journal of Science and Medicine in Sport, 13, e30-e31. doi: 10.1016/j.jsams.2010.10.525 
Christmann, J., Akamphuber, M., Müllenbach, A.L., \& Güllich, A. (2018). Crunch time in the NBA-The effectiveness of different play types in the endgame of close matches in professional basketball. International Journal of Sports Science Coaching, 13(6), 1090-1099. doi.org/10.1177/1747954118772485

Conte, D., Favero, T.G., Niederhausen, M., Capranica, L., \& Tessitore, A. (2016). Effect of different number of players and training regimes on physiological and technical demands of ball-drills in basketball. Journal of Sports Sciences, 34(8), 780-786. doi: 10.1080/02640414.2015.1069384

Conte, D., Favero, T.G., Niederhausen, M., Capranica, L., \& Tessitore, A. (2017). Determinants of the effectiveness of fast break actions in elite and sub-elite Italian men's basketball games. Biology of Sport, 34(2), 177-183. doi: 10.5114 /biolsport.2017.65337

Conte, D., Scanlan, A.T., Dalbo, V.J., Gang, S.Z., Smith, M.R., Bietkis, T., \& Matulaitis, K. (2020). Dribble deficit quantifies dribbling speed independently of sprinting speed and differentiates between age categories in pre-adolescent basketball players. Biology of Sport, 37(3), 261-267. doi: 10.5114/biolsport.2020.95637

Cortes, N., Onate, J., \& Morrison, S. (2014). Differential effects of fatigue on movement variability. Gait and Posture, 39(3), 888-893. doi: 10.1016/j.gaitpost.2013.11.020

Cortis, C., Tessitore, A., Lupo, C., Pesce, C., Fossile, E., Figura, F., \& Capranica, L. (2011). Inter-limb coordination, strength, jump, and sprint performances following a youth men's basketball game. Journal of Strength and Conditioning Research, 25(1), 135-142. doi: 10.1519/JSC.0b013e3181bde2ec

Dal Pupo, J., Detanico, D., Ache-Dias, J., \& Santos, S.G. (2017). The fatigue effect of a simulated futsal match protocol on sprint performance and kinematics of the lower limbs. Journal of Sports Sciences, 35(1), 81-88. doi: $10.1080 / 02640414.2016 .1156727$

Dos Santos, F.G., Pacheco, M.M., Basso, L., Bastos, F.H., \& Tani, G. (2020). Development and validation of a checklist to assess proficient performance of basketball straight speed dribbling skill. Journal of Human Kinetics, 71, 21-31. doi: 10.2478/hukin-2019-0073

Doyle, B., Browne, D., \& Horan, D. (2020). The relationship of aerobic endurance and linear speed on repeat sprint ability performance in female international footballers. International Journal of Human Movement and Sports Sciences, 8, 147-153. doi: 10.13189/saj.2020.080407

Erčulj, F., Blas, M., \& Bračič, M. (2010). Physical demands on young elite European female basketball players with special reference to speed, agility, explosive strength, and take-off power. Journal of Strength and Conditioning Research, 24(11), 2970-2978. doi: 10.1519/JSC.0b013e3181e38107

Erculj, F., \& Supej, M. (2009). Impact of fatigue on the position of the release arm and shoulder girdle over a longer shooting distance for an elite basketball player. Journal of Strength and Conditioning Research, 23(3), 10291036. doi: 10.1519/JSC.0b013e3181a07a27

Erčulj, F., \& Supej, M. (2006). The impact of fatigue on jump shot height and accuracy over a longer shooting distance in basketball. Baltic Journal of Sport and Health Sciences, 63(4), 35-41.

Evangelos, T., Alexandros, K., \& Nikolaos, A. (2005). Analysis of fast breaks in basketball. International Journal of Performance Analysis in Sport, 5(2), 17-22. doi: 10.1080/24748668.2005.11868324

Fujii, K., Yamada, Y., \& Oda, S. (2010). Skilled basketball players rotate their shoulders more during running while dribbling. Perceptual and Motor Skills, 110(3; Pt 1), 983-994. doi: 10.2466/pms.110.3.983-994

Guimarães, E., Baxter-Jones, A., Maia, J., Fonseca, P., Santos, A., Santos, E., Janeira, M.A. (2019). The roles of growth, maturation, physical fitness, and technical skills on selection for a Portuguese under-14 years basketball team. Sports (Basel), 7(3), 61. doi: 10.3390/sports7030061

Hinde, K., White, G., \& Armstrong, N. (2021). Wearable devices suitable for monitoring twenty four hour heart rate variability in military populations. Sensors (Basel), 21, 1061. doi: 10.3390/s21041061

Jakovljević, S., Karalejić, M., Ivanović, J., Štrumbelj, E., \& Erčulj, F. (2017). Efficiency of speed and agility dribbling of young basketball players. Kinesiologia Slovenica, 23(2), 22-32.

Janicijevic, D., Knezevic, O.M., Mirkov, D.M., Pérez-Castilla, A., Petrovic, M., Samozino, P., \& Garcia-Ramos, A. (2020). Assessment of the force-velocity relationship during vertical jumps: Influence of the starting position, analysis procedures and number of loads. European Journal of Sport Science, 20(5), 614-623. doi: 10.1080/17461391.2019.1645886

Krause, J.V., \& Nelson, C. (2018). Basketball skills and drills. Champaign, IL: Human Kinetics.

Lessi, G.C., dos Santos, A.F., Batista, L.F., de Oliveira, G.C., \& Serrão, F.V. (2017). Effects of fatigue on lower limb, pelvis and trunk kinematics and muscle activation: Gender differences. Journal of Electromyography and Kinesiology, 32, 9-14. doi: 10.1016/j.jelekin.2016.11.001

Lessi, G.C., \& Serrão, F.V. (2017). Effects of fatigue on lower limb, pelvis and trunk kinematics and lower limb muscle activity during single-leg landing after anterior cruciate ligament reconstruction. Knee Surgery, Sports Traumatology, Arthroscopy, 25(8), 2550-2558. doi: 10.1007/s00167-015-3762-x

Li, F., Knjaz, D., \& Rupčić, T. (2021). Influence of fatigue on some kinematic parameters of basketball passing. International Journal of Environmental Research and Public Health, 18(2), 700. doi: 10.3390/ijerph18020700

Lyons, M., Al-Nakeeb, Y., \& Nevill, A. (2006). The impact of moderate and high intensity total body fatigue on passing accuracy in expert and novice basketball players. Journal of Sports Science and Medicine, 5(2), 215. 
Mancha-Triguero, D., García-Rubio, J., Calleja-González, J., \& Ibáñez, S.J. (2019). Physical fitness in basketball players: A systematic review. Journal of Sports Medicine and Physical Fitness, 59(9), 1513-1525. doi: 10.23736/ s0022-4707.19.09180-1

Mancha-Triguero, D., García-Rubio, J., Gamonales, J.M., \& Ibáñez, S.J. (2021). Strength and speed profiles based on age and sex differences in young basketball players. International Journal of Environmental Research and Public Health, 18(2), 643. doi: 10.3390/ijerph18020643

Matulaitis, K., \& Bietkis, T. (2021). Prediction of offensive possession ends in elite basketball teams. International Journal of Environmental Research and Public Health, 18(3)1083. doi: 10.3390/ijerph18031083

McInnes, S.E., Carlson, J.S., Jones, C.J., \& McKenna, M.J. (1995). The physiological load imposed on basketball players during competition. Journal of Sports Sciences, 13(5), 387-397. doi: 10.1080/02640419508732254

Milanović, D., Selmanović, A., \& Škegro, D. (2014). Characteristics and differences of basic types of offenses in European and American top-level basketball. In D. Milanović \& G. Sporiš (Eds.), Proceedings Book of the 7th International Scientific Conference on Kinesiology (pp. 400-403). Zagreb: Faculty of Kinesiology, University of Zagreb.

Morin, J.-B., Samozino, P., Edouard, P., \& Tomazin, K. (2011). Effect of fatigue on force production and force application technique during repeated sprints. Journal of Biomechanics, 44(15), 2719-2723. doi: 10.1016/j. jbiomech.2011.07.020

Mulazimoglu, O., Yanar, S., Tunca Evcil, A., \& Duvan, A. (2017). Examining the effect of fatigue on shooting accuracy in young basketball players. The Anthropologist, 27(1-3), 77-80. doi: 10.1080/09720073.2017.1311671

Nakano, N., Fukashiro, S., \& Yoshioka, S. (2020). The effect of increased shooting distance on energy flow in basketball jump shot. Sports Biomechanics, 19(3), 366-381. doi: 10.1080/14763141.2018.1480728

Okazaki, V.H.A., \& Rodacki, A.L.F. (2012). Increased distance of shooting on basketball jump shot. Journal of Sports Science and Medicine, 11(2), 231.

Okubo, H., \& Hubbard, M. (2015). Kinematics of arm joint motions in basketball shooting. Procedia Engineering, 112, 443-448. doi: 10.1016/j.proeng.2015.07.222

Robalo, R.A.M., Diniz, A., Fernandes, O., \& Passos, P.J.M. (2021). The role of variability in the control of the basketball dribble under different perceptual setups. European Journal of Sport Science, 21(4), 521-530. doi: $10.1080 / 17461391.2020 .1759695$

Robert-Lachaine, X., Mecheri, H., Larue, C., \& Plamondon, A. (2017). Validation of inertial measurement units with an optoelectronic system for whole-body motion analysis. Medical and Biological Engineering and Computing, 55(4), 609-619. doi: 10.1007/s11517-016-1537-2

Rupčić, T., Feng, L., Matković, B.R., Knjaz, D., Dukarić, V., Baković, M., \& Garafolić, H. (2020). The impact of progressive physiological loads on angular velocities during shooting in basketball—Case study. Acta Kinesiologica, 14(2), 102-109.

Scanlan, A., Dascombe, B., \& Reaburn, P. (2011). A comparison of the activity demands of elite and sub-elite Australian men's basketball competition. Journal of Sports Sciences, 29(11), 1153-1160. doi: 10.1080/02640414.2011.582509

Scanlan, A.T., Dalbo, V.J., Conte, D., Stojanović, E., Stojiljković, N., Stanković, R., Milanović, Z. (2019). Caffeine supplementation has no effect on dribbling speed in elite basketball players. International Journal of Sports Physiology and Performance, 14(7), 997-1000. doi: 10.1123/ijspp.2018-0871

Scanlan, A.T., Dascombe, B.J., Kidcaff, A.P., Peucker, J.L., \& Dalbo, V.J. (2015). Gender-specific activity demands experienced during semiprofessional basketball game play. International Journal of Sports Physiology and Performance, 10(5), 618-625. doi: 10.1123/ijspp.2014-0407

Sekulic, D., Pehar, M., Krolo, A., Spasic, M., Uljevic, O., Calleja-González, J., \& Sattler, T. (2017). Evaluation of basketball-specific agility: Applicability of preplanned and nonplanned agility performances for differentiating playing positions and playing levels. Journal of Strength and Conditioning Research, 31(8), 2278-2288. doi: 10.1519/jsc.0000000000001646

Slawinski, J.P., Louis, J., Poli, J., Tiollier, E., Khazoom, C., \& Dinu, D. (2018). The effects of repeated sprints on the kinematics of 3-point shooting in basketball. Journal of Human Kinetics, 62, 5-14. doi: 10.1515/hukin-2017-0156

Sorrentino, I., Andrade Chavez, F.J., Latella, C., Fiorio, L., Traversaro, S., Rapetti, L., Pucci, D. (2020). A novel sensorised insole for sensing feet pressure distributions. Sensors (Basel), 20(3), 747. doi: 10.3390/s20030747

Speer, K.E., Semple, S., Naumovski, N., \& Mckune, A.J. (2020). Measuring heart rate variability using commercially available devices in healthy children: A validity and reliability study. European Journal of Investigation in Health, Psychology, Education, 10, 390-404. doi: 10.3390/ejihpe10010029

Spiteri, T., Cochrane, J.L., Hart, N.H., Haff, G.G., \& Nimphius, S. (2013). Effect of strength on plant foot kinetics and kinematics during a change of direction task. European Journal of Sport Science, 13(6), 646-652. doi: $10.1080 / 17461391.2013 .774053$

Spiteri, T., Nimphius, S., Hart, N.H., Specos, C., Sheppard, J.M., \& Newton, R.U. (2014). Contribution of strength characteristics to change of direction and agility performance in female basketball athletes. Journal of Strength and Conditioning Research, 28(9), 2415-2423. doi: 10.1519/jsc.0000000000000547

Sporiš, G., Vučetić, V., Milanović, L., Milanović, Z., Krespi, M., \& Krakan, I. (2014). A comparison anaerobic endurance capacity in elite soccer, handball and basketball players. Kinesiology, 46(Supp 1), 52-59. 
Stricker, G., Scheiber, P., Lindenhofer, E., \& Müller, E. (2010). Determination of forces in alpine skiing and snowboarding: Validation of a mobile data acquisition system. European Journal of Sport Science, 10(1), 31-41. doi: 10.1080/17461390903108141

Tanner, R.K., Fuller, K.L., \& Ross, M.L. (2010). Evaluation of three portable blood lactate analysers: Lactate Pro, Lactate Scout and Lactate Plus. European Journal of Applied Physiology, 109, 551-559. doi: 10.1007/s00421-010-1379-9

Thorpe, R.T., Atkinson, G., Drust, B., \& Gregson, W. (2017). Monitoring fatigue status in elite team-sport athletes: Implications for practice. International Journal of Sports Physiology and Performance, 12(Suppl 2), S227S234. doi: 10.1123/ijspp.2016-0434

Torres-Ronda, L., Ric, A., Llabres-Torres, I., de Las Heras, B., \& Schelling, I.D.A.X. (2016). Position-dependent cardiovascular response and time-motion analysis during training drills and friendly matches in elite male basketball players. Journal of Strength and Conditioning Research, 30(1), 60-70. doi: 10.1519/jsc.0000000000001043

Uygur, M., Goktepe, A., Ak, E., Karabörk, H., \& Korkusuz, F. (2010). The effect of fatigue on the kinematics of free throw shooting in basketball. Journal of Human Kinetics, 24, 51-56.

Wan, J.J., Qin, Z., Wang, P.Y., Sun, Y., \& Liu, X. (2017). Muscle fatigue: General understanding and treatment. Experimental and Molecular Medicine, 49(10), e384. doi: 10.1038/emm.2017.194

World Medical Association. (2013). Declaration of Helsinki: Ethical principles for medical research involving human subjects. JAMA, 310(20), 2191-2194. doi: 10.1001/jama.2013.281053

Zivkovic, M.Z., Djuric, S., Cuk, I., Suzovic, D., \& Jaric, S. (2017). Muscle force-velocity relationships observed in four different functional tests. Journal of Human Kinetics, 56, 39-49. doi: 10.1515/hukin-2017-0021

Submitted: May 13, 2021

Accepted: November 6, 2021

Published Online First: December 9, 2021

Correspondence to:

Feng Li, Ph.D.

Laboratory for Sports Games, Faculty of Kinesiology,

University of Zagreb

Horvaćanski zavoj 15, Zagreb, Croatia

Phone: +385953658798

E-mail: li.feng@kif.unizg.hr

\section{Ethical Approval Information}

The study was conducted according to the guidelines of the Declaration of Helsinki and approved by the Ethics Committee of the Faculty of Kinesiology, University of Zagreb (ethical code 108/2020, November 27, 2020).

\section{Funding}

The first author was supported by the China Scholarship Council (CSC) from the Ministry of Education of P.R. China under Grant [(2019) 110]. 